\title{
Infrared Lateral Photoconductivity of InGaAs Quantum Dots: the Temperature Dependence
}

\author{
L.D. Moldavskaya*, V.I. Shashkin, M.N. Drozdov, \\ V.M. Daniltsev, A.V. Antonov and A.N. Yablonsky \\ Institute for Physics of Microstructures, Russian Academy of Sciences \\ Nizhni Novgorod, Russia
}

\begin{abstract}
We report the temperature dependence of lateral infrared photoconductivity in multilayer InGaAs/GaAs heterostructures with selectively doped quantum dots fabricated by metalorganic chemical vapor deposition. Two spectral lines of normal-incidence intersubband photoconductivity (90 meV and $230 \mathrm{meV}$ ) and a line originating from interband transitions $(930 \mathrm{meV})$ were observed. The photoconductivity line $230 \mathrm{meV}$ is revealed up to the temperature $140 \mathrm{~K}$. The long-wavelength photoconductivity line $90 \mathrm{meV}$ is quenched rapidly at the temperature $30 \div 40 \mathrm{~K}$ owing to redistribution of photoexcited carriers between small and large dots. The obtained results confirm the hypothesis about bimodal distribution of quantum dot sizes.
\end{abstract}

PACS numbers: 78.30.Fs, 78.55.Cr, 78.66.Fd

\section{Introduction}

Zero-dimensional quantum dot (QD) nanostructures have attracted an increasing attention in the last years due to their unique properties and potential device applications, such as photodetectors and lasers. Quantum dot photodetectors have several advantages over quantum wells photodetectors, in particular, high lifetime of photoexcited electrons due to reduced phonon scattering (phonon bottleneck effect) [1,2]. This provides potentially more efficient detection and high-temperature operation. Furthermore, unlike quantum wells, QDs are sensitive to normally incident radiation owing to relax polarisation selection rule. Primarily photoconductivity (PC) of QDs heterostructures was investigated with a vertical electron transport. It was revealed that InGaAs/GaAs QD structures de-

*corresponding author; e-mail: lmd@ipm.sci-nnov.ru 
tect mid- and far IR radiation $(4-20 \mu \mathrm{m})[2-8]$. Later, it was shown that a lateral carrier transport can result in the improvement of the device performance, which is attributed to the longer carrier lifetime as compared to vertical one [9, 10]. In such a case, the carrier transport occurs along the wetting layer or the neighboring channel with high electron mobility. Recently we have reported the pilot experiments with lateral intersubband photoconductive multilayer InGaAs/GaAs heterostructures with selectively doped QDs fabricated by metalorganic chemical vapor deposition (MOCVD) [11]. In this paper we present the temperature dependence $(T=8 \div 150 \mathrm{~K}$ ) of lateral infrared photoconductivity.

Optical properties of QDs depend on their size and shape, which can be varied by their growth conditions. Atomic force microscopy (AFM) is known as an informative method for investigation of QD arranged on surface. However, overgrowth of QDs with a semiconductor layer results in changes of their lateral sizes and height, composition and surface density due to partial dissolving of QDs in cap-layer and rise non-uniform strain fields around them. Transmission electron microscopy (TEM) is one of main methods of characterization of buried QDs, but non-uniform strain around QDs and wetting layer leads to considerable errors of measurements. Therefore it has been suggested to reconstruct sizes of overgrown QDs by deconvolution of sizes from energy levels found from measurements of photoluminescence spectra [12]. In Refs. [13, 14] the authors propose to use the temperature dependence of dark current and IR absorption in order to take account of nonradiative transitions for randomly distributed sizes of QDs. In this work it is shown that temperature dependence of photoconductivity also allows to determine nonradiative transitions and to analyse QD sizes distribution.

\section{Experiment}

The InGaAs/GaAs QDs were grown in low pressure MOCVD reactor on semi-insulating (100) GaAs substrates. An active region had 10 layers of InGaAs QDs separated by $90 \mathrm{~nm}$ of GaAs. The $\delta$-doped with Si layers were $2.5 \mathrm{~nm}$ beneath the QD layers. The QD layers and thin GaAs cap layers were grown at $480^{\circ} \mathrm{C}$, the $90 \mathrm{~nm}$ GaAs barrier layers were grown at $600^{\circ} \mathrm{C}$ after flushing the sources and interrupting the growth. X-ray diffraction, photoluminescence, atomic force and transmission electron microscopy were applied to find the optimal growth condition and to characterize multilayer QD structures. According to TEM cross-section measurements the QDs have a lens shape geometry with a typical height of $2.5-3 \mathrm{~nm}$ and a lateral size of $20 \mathrm{~nm}$. Height histogram obtained by AFM on the reference samples (with QDs on the surface) shows two typical values $(4 \mathrm{~nm}$ and $6 \mathrm{~nm}$ ). Lateral size to height ratio $(k)$ of QDs placed on surface is equal to 3-4. Electron and energy spectra of such QD can be calculated accordingly to [15]. The QDs have a few excited electron states. On the other hand, TEM cross-section shows buried QDs with a sufficiently low height $2.5-3 \mathrm{~nm}$ and $k=7-8$. As the authors 
of [14] we assume that during the growth of GaAs over-layer adatoms at the QD apex migrate to the side of dots and their height reduces. Lens shaped QD with a lateral size of $20 \mathrm{~nm}$ has only ground electron level.

Photoresponse measurements were performed for normal incident light with lateral electron transport. Photoconductive spectra were obtained using Fourier spectrometer BOMEM with a globar source.

\section{Results}

Figures 1 and 2 display the results of the measurements. Figure 1a,b shows the temperature dependence of the $\mathrm{PC}$ spectrum in the two spectral regions obtained using KRS and $\mathrm{CaF}_{2}$ filters. Two spectral lines of intersubband PC (90 meV and $230 \mathrm{meV}$ ) and a line originating from interband transitions $(930 \mathrm{meV})$ were observed. The temperature of measurements was varied from $8 \mathrm{~K}$ to $150 \mathrm{~K}$. Peaks value of these $\mathrm{PC}$ lines as a function of temperature is shown in Fig. 2. The long-wavelength line $90 \mathrm{meV}(\lambda=14 \mu \mathrm{m})$ was the most intensive. This signal disappears when the temperature was increased up to $30-40 \mathrm{~K}$ because of thermal escape of charge carriers out of the shallow levels. The additional mechanism of quenching is discussed below. The complicated form of the spectral response curves in these regions may be caused by an appreciable influence of optical phonons as it is supposed in $[16,17]$.
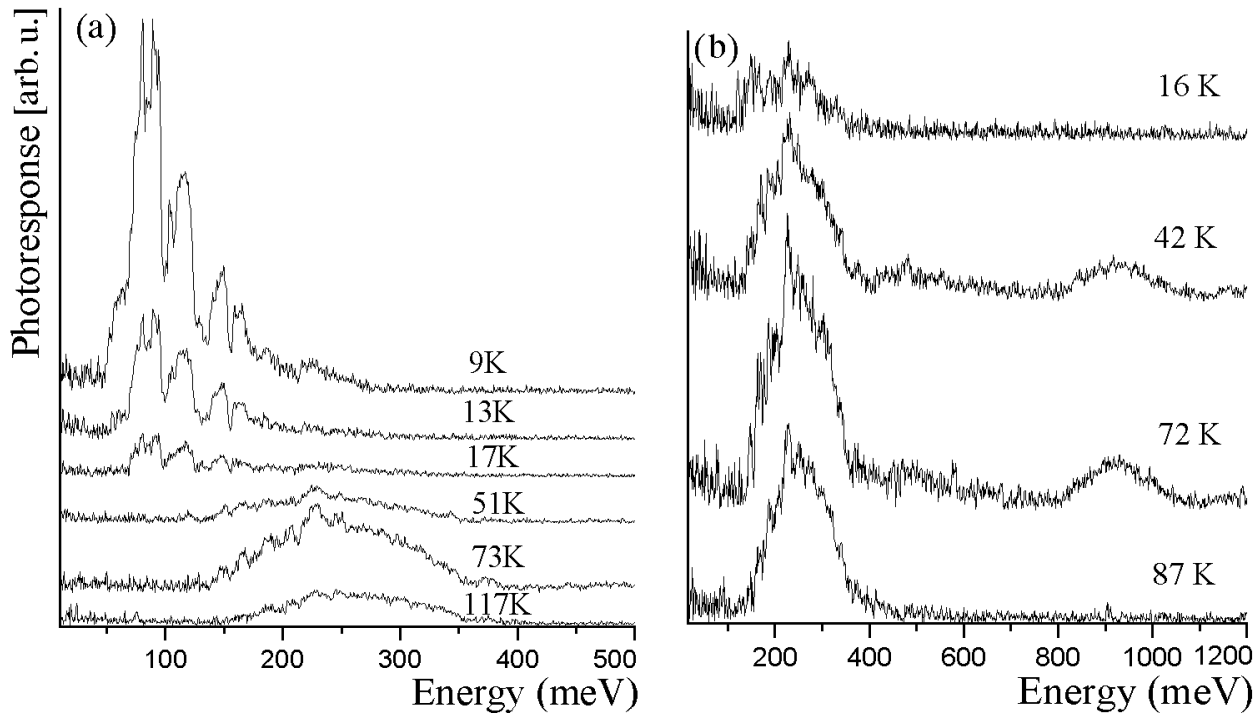

Fig. 1. PC spectra at different temperatures measured with filters: (a) KRS-5 (in the range from $40 \mathrm{meV}$ to $500 \mathrm{meV}$ transmission is equal to $65 \div 70 \%$ ); (b) $\mathrm{CaF}_{2}$ (in the range from $180 \mathrm{meV}$ to $1200 \mathrm{meV}$ transmission is equal to $95 \%$ ). 


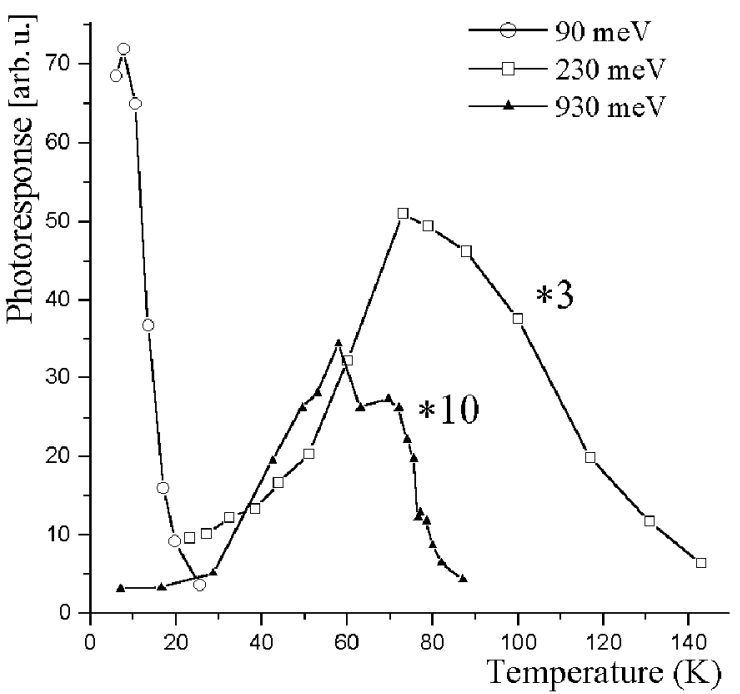

Fig. 2. Maximum values of the three PC lines as a function of temperature.

PC signal in the region $230 \mathrm{meV}(\lambda=5.4 \mu \mathrm{m})$ was observed in temperature range from $30 \mathrm{~K}$ to $140 \mathrm{~K}$. Two similar groups of PC lines $(80 \mathrm{meV}$ and $180 \mathrm{meV}$ ) was found in [16] for multilayer structures with QDs with a base diameter of $18 \mathrm{~nm}$ and height of $2.5 \mathrm{~nm}$. Authors of [16] connected it with the three confinement levels in QD and additional influence of $S$ - and $P$-polarization of incident radiation. At the same time Fig. 2 shows explicit thermo-activated behavior of the PC line at $230 \mathrm{meV}$. At temperatures below $30 \mathrm{~K}$ the signal is absent, this testifies that deeper levels are not occupied. In our opinion these data confirm the assumption of our work [11] about bimodal distribution of QD sizes in the structures based on technological growth conditions. The similar bimodal distribution was reported in $[12,18]$. Small QDs determines long-wavelength signal at $90 \mathrm{meV}$. The large QDs are apparently separated by additional energy barrier of about $2-3 \mathrm{meV}$ (corresponding to the temperature $30 \mathrm{~K}$ ), therefore their occupation has the thermo-stimulated behavior and they are not occupied at low temperature. It can be noted that TEM cross-section shows that QD image contrast is almost symmetric relatively to wetting layer. It is probably owing to the elastic strain of GaAs layer around the QDs [15]. At present we cannot determine the difference of strain value around the small and large QDs. We believe that the strain of GaAs layer is just the most probable reason of occurrence of the $2-3 \mathrm{meV}$ barrier. At temperature above $30 \mathrm{~K}$ electrons overcome this barrier and occupy deep levels in the large dots causing PC signal in region near $230 \mathrm{meV}$. It should be noticed that thermo-activated mechanism of the occupation of the states in the large dots leads to competing of occupation of dots with different sizes in these temperature ranges. Small QDs (and shallow levels) are getting empty quickly. As a result the 
long-wavelength PC line $90 \mathrm{meV}$ is quenched rapidly at the temperature $30-40 \mathrm{~K}$ as is seen at Fig. 2 .

The shortest-wavelength line in the IR PC spectrum in Fig. $2 \mathrm{~b}$ about $930 \mathrm{meV}$ $(1.33 \mu \mathrm{m})$ originates from interband transitions in large QDs. The temperature dependence of this line has activated behavior with the same activated temperature as for the line at $230 \mathrm{meV}$. Photoexcited electrons have to overcome the same barrier 2-3 meV to get state in wetting layer or GaAs barrier layer. The quenching of $\mathrm{PC}$ line $930 \mathrm{meV}$ occurs in a narrow temperature range near $80 \mathrm{~K}$. In our opinion it can be caused by strong increase in recombination rate for interband transitions when temperature is above $80 \mathrm{~K}$ and quick decrease in lifetime of photoexcited carriers. It is known that narrow-band semiconductor photodetectors have fundamental limitation on working temperatures about $80 \mathrm{~K}$ owing to the Auger recombination. In the case of intraband transitions in QDs there is no this limitation as can be seen in Fig. 2.

\section{Summary}

We have studied the temperature dependence of lateral infrared photoconductivity in multilayer InGaAs/GaAs heterostructures with selectively doped quantum dots. Two spectral lines of normal-incidence intersubband PC (90 meV and $230 \mathrm{meV}$ ) and a line originating from interband transitions $(930 \mathrm{meV})$ were observed. Thermo-activated behavior of IR PC was observed for lines $230 \mathrm{meV}$ and $930 \mathrm{meV}$. It can be reasonably explained by a bimodal distribution of dot sizes and existence of energy barrier for large dots. In our opinion, this barrier rises due to strain around QDs. Thermo-activated mechanism of the occupation of the states in the large dots result in competing population of deep and shallow levels. As a result the long-wavelength $\mathrm{PC}$ line $90 \mathrm{meV}$ is quenched rapidly at the temperature $30-40 \mathrm{~K}$. The obtained results demonstrate also that temperature dependence of photoconductivity allows to determine nonradiative transitions and to analyse QD size distribution.

\section{Acknowledgment}

This work was supported in part by RFBR grants No. 01-02-16451, 03-0216780, 03-02-17404 and by the program "Physics of Solid State Nanostructures".

\section{References}

[1] S.-F. Tang, S.-Y. Lin, S.-C. Lee, Appl. Phys. Lett. 78, 2428 (2001).

[2] E. Finkman, S. Maimon, V. Immer, G. Bahir, S.E. Schacham, F. Fossard, F.H. Julien, J. Brault, M. Gedry, Phys. Rev. B 63, 045323 (2001).

[3] S. Maimon, E. Finkman, G. Bahir, S.E. Schacham, J.M. Garsia, P.M. Petroff, Appl. Phys. Lett. 73, 2003 (1998). 
[4] H.C. Liu, M. Gao, J. Mc Caffey, Z.R. Wasilewski, S. Fafard, Appl. Phys. Lett. 78, 79 (2001).

[5] D. Pan, E. Towe, S. Kennerly, Appl. Phys. Lett. 75, 2719 (1999).

[6] D. Pan, E. Towe, S. Kennerly, Appl. Phys. Lett. 73, 1937 (1998).

[7] S. Kim, H. Mohseni, M. Erdtmann, E. Michel, C. Jelen, M. Razedhi, Appl. Phys. Lett. 73, 963 (1998).

[8] J. Phillips, K. Kamath, P. Bhattacharya, Appl. Phys. Lett. 72, 2020 (1998).

[9] S.-W. Lee, K. Hirakawa, Y. Shimada, Physica E 7, 499 (2000).

[10] L. Chu, A. Zenner, M. Bichter, G. Abstreiter, Appl. Phys. Lett. 79, 2249 (2001).

[11] L.D. Moldavskaya, V.I. Shashkin, M.N. Drozdov, Y.N. Drozdov, V.M. Daniltsev, A.V. Murel, B.A. Andreev, A.N. Yablonsky, S.A. Gusev, D.M. Gaponova, Physica E 17, 634 (2003).

[12] G. Saint-Girons, I. Sagnes, J. Appl. Phys. 91, 10115 (2002).

[13] Y.H. Kang, J. Park, U.H. Lee, S. Hong, Appl. Phys. Lett. 82, 1099 (2003).

[14] D. Pal, D. Firsov, E. Towe, Physica E 15, 6 (2002).

[15] M. Grundmann, O. Stier, D. Bimberg, Phys. Rev. B 52, 11969 (1995).

[16] B. Aslan, H.C. Liu, M. Korkusinski, S.-J. Cheng, P. Hawrylak, Appl. Phys. Lett. 82, 630 (2003).

[17] J.J. Finley, M. Skalitz, M. Arzberger, A. Zrenner, G. Bohm, G. Abstreiter, Appl. Phys. Lett. 73, 2618 (2003).

[18] E.-T. Kim, Z. Chen, A. Madhukar, Appl. Phys. Lett. 79, 3341 (2001). 